\title{
Multifragmentation - what the data tell us about the different models
}

\author{
Regina Nebauer ${ }^{1,2}$, Jörg Aichelin ${ }^{1}$ a \\ ${ }^{\text {a1SUBATECH }}$ \\ Université de Nantes, EMN, IN2P3/CNRS \\ 4, Rue Alfred Kastler, 44070 Nantes Cedex 03, France \\ ${ }^{2}$ Institute for Theoretical Physics Universität Rostock, Rostock, Germany.
}

We discuss what the presently collected data tell us about the mechanism of multifragmentation by comparing the results of two different models, which assume or show an opposite reaction scenario, with the recent high statistics $4 \pi$ experiments performed by the INDRA collaboration. We find that the statistical multifragmentation model and the dynamical Quantum Molecular Dynamics approach produce almost the same results and agree both quite well with experiment. We discuss which observables may serve to overcome this deadlock on the quest for the reaction mechanism. Finally we proof that even if the system is in equilibrium, the fluctuation of the temperature due to the smallness of the system renders the caloric curve useless for the proof of a first order phase transition.

\section{Models for describing multifragmentation}

About 15 years ago it has been found that in nucleus-nucleus collisions at intermediate energies up to 15 intermediate mass fragments (IMF's) with $Z \geq 3$ are created. Since then many dedicated experiments have been performed to "nail down" the reaction mechanism but despite of all experimental and theoretical efforts we have not found an answer yet. The different contributions in these proceedings present a clear evidence for this fact. Over the years two different conjectures have been launched and developed, improved and further improved without giving a conclusive answer. The reason is, as we will see, that the two most advanced but also almost opposite approaches predict the same behavior for several key observables. Before we discuss this in detail we will introduce shortly the two principal approaches.

\subsection{Multifragmentation as a statistical process}

One may assume that multifragmentation is a statistical process. This means that during the reaction at least a subsystem comes to statistical equilibrium and maintains this equilibrium during the expansion until it has reached the freeze out volume. Then it disintegrates INSTANTANEOUSLY into neutrons, light charged particles (LCP's) and IMF's. Because it is assumed that at freeze out the system is in statistical equilibrium the disintegration pattern is determined by phase space. This means that one searches for all microstates (consisting of fragments and nucleons with a given kinetic and excitation energy) which are compatible with a total energy, a total proton and neutron number 
and a given volume. Each microstate has the same weight and hence one samples all

microstates in order to obtain $\frac{d^{4} \sigma}{d^{3} p d A}$. There are three different models ([1] - [3]) which perform this task. Sequential decay (i.e. the formation of a compound nucleus which decays by subsequent fragment emission) has been ruled out because it gives too few fragments.

Unfortunately this task is not as easy as it seems to be, both conceptually and numerically. Therefore the results of these models differ considerable despite of the fact that one is tempted to believe that counting the number of microstates is a well defined task.

Conceptually there are two problems:

a) there is no theory which tells us how to treat the unstable states. Usually the number of excited states of fragments is calculated via a level density parameter but some of these excited states are unstable against particle (usually neutron) emission. Therefore it is not evident, whether they should be treated as an excited fragment $\mathrm{A}$ or as a fragment A-1 plus one neutron. Both descriptions give rise to different microstates. The different answers to this problem yield a quite different neutron yield as has been recently found out by Toeke and Schroeder 《4.

b) the freeze out volume can be defined quite differently. Whereas in ref [2] a constant freeze out volume is assumed in ref. [1] the freeze out volume depends on the microstate. There is no convincing argument why the one assumption should be better than the other.

Also numerically (especially concerning the Coulomb energy) the approaches differ considerably. It is the merit of Gross and Sneppen [5] to have discussed all the differences in detail and to have demonstrated how it comes that two seemingly identical approaches produce quite different results.

\subsection{Multifragmentation as a dynamical process}

On the other hand there are dynamical models. Originally developed to describe proton spectra, particle production and the influence of the nuclear equation of state on observables like the flow, they have later been further developed to describe multifragmentation as well. In 1985 the Quantum Molecular Dynamics model [6,7] has been introduced which describes the time evolution of the n-body Wignerdensity and hence allows for the investigation of fragments which are n-body correlations in this context. In this approach the nucleons are presented as Gaussian wave functions

$\phi_{\alpha}\left(p_{1}, t\right)=\left(\frac{L}{2 \pi}\right)^{3 / 4} e^{-\left(p_{1}-p_{\alpha}\right)^{2} L / 4} e^{-i x_{\alpha} p_{1}} e^{-2 i p_{1} p_{\alpha} t / 2 m+i p_{\alpha}^{2} t / 2 m}$

where $\alpha$ characterizes the nucleon. The time evolution of the individual nucleons is determined by a variational principle. At the very end a minimum spanning tree is applied to identify the clusters. In these models the whole reaction, starting from the initially separated projectile and target nuclei until the finally observable fragments and single particles is simulated. Besides the Hamiltonian which is, however, well determined by the requirement to have stable nuclei with the right binding energy, the only parameters to change are the impact parameter and the beam energy. The different final states, created in each individual simulation, are caused by the different possibilities to realize the same single particle distribution and the same binding energy by choosing different positions $x_{\alpha}$ and momenta $p_{\alpha}$ for the individual nucleons. For details of this model we refer to the references [6,7]. 
There are two conceptual criticisms of this model. First, that the wave function of the nucleons is not antisymmetrized. Therefore one argued, making reference to a Fermi gas, that the specific heat will be different as compared to an antisymmetrized system. Hence, if the system comes to equilibrium one expected a quite different fragmentation pattern. Although this is true of course for a Fermi gas, the Aladin collaboration has found [8] that this is not true for an interacting system containing nucleons and fragments. The above mentioned incertainties of the statistical models have a larger influence on the fragmentation pattern than the different specific heats. Second, being a semiclassical approach, the fragments have the properties of the Weizsäcker mass formula but are not real quantum states. In addition effective charges are used. Hence isotopic distributions cannot be calculated.

Nevertheless with two parameters which have to be adjusted, the width of the wave function and the fragmentation radius of the minimum spanning tree, the QMD model has described many low energy fragmentation data [6,9]. At these energies the whole nucleus disintegrates into fragments. At higher beam energies, where only the weakly excited spectator matter disintegrates into fragments the model in its original version failed [10]. Only recently the reason for this failure has been identified and the model describes now spectator fragmentation as well [11].

Careful investigations have shown that in these simulation programs the system passes never a state of equilibrium [12]. In almost all cases where multifragmentation has been observed the systems is, on the contrary, far away from it. This can easily be seen from the videos which have been produced with help if this program [13].

\section{Multifragmentation - a theory invariant phenomenon?}

In view of the quite different dynamics assumed or obtained in these both approaches to multifragmentation it seems to be astonishing that experiments have not yet decided which of the both conjectures is correct. This fact becomes more understandable if one considers in similar but somewhat simpler models, what one expects to see. For this purpose we assume that the system can be described by canonical thermodynamics, although this is not the case, as we will see later. For the question discussed here it serves the purpose.

First we will suppose a fast fragmentation process as it is the case for a dynamical process. If the disintegration of the nucleus is instantaneous each nucleon keeps its momentum due to the Fermi motion and one expects an average fragment kinetic energy of $3 / 5 E_{F}$ independent of the fragment size [14, where $E_{F}$ is the Fermi energy. The same independence one expects if the fragments are formed very late, after the system has been expanded while maintaining thermal equilibrium as assumed in the statistical model. This requires the opposite, that the disintegration is very slow. Here the average kinetic energy is $3 / 2 \mathrm{~T}$, where $\mathrm{T}$ is the temperature at freeze out. Hence it is only the absolute value but not the mass dependence of the mean kinetic energy which is different.

The same ambiguity one finds for the mass yield. Fischer [15] has pointed out that finite systems close to a phase transition show a fragment distribution $\sigma(A) \propto A^{\tau}$. But this power law dependence appears in many physical processes which are not thermal at all [16 18. Figure 11 shows schematically this behavior.

This ambiguity remains valid if one employs the more sophisticated models discussed 


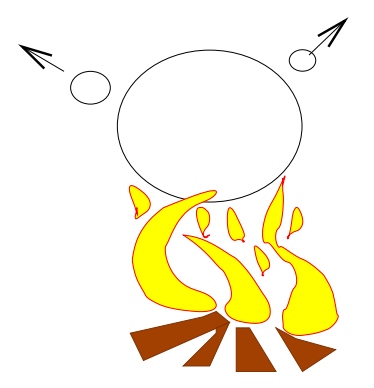

statistical decay

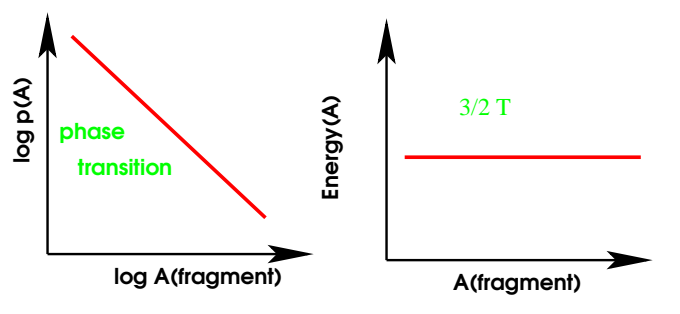

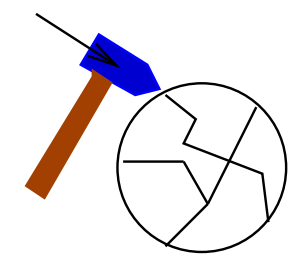

dynamical fragmentation
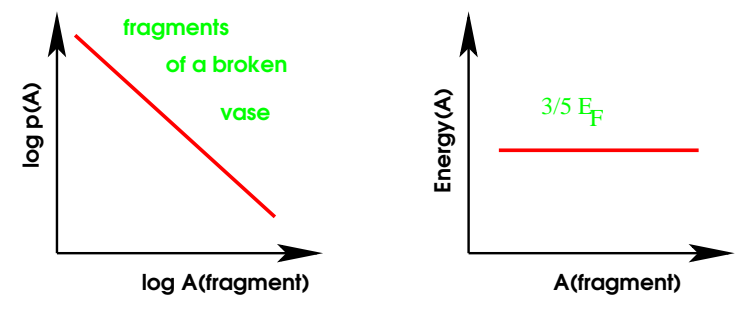

Figure 1. Similarity of the results one expects to see independent of whether multifragmentation is a statistical or a dynamical process.

above. For our studies we use data and simulations for central collisions of the reaction $50 \mathrm{AMeV} \mathrm{Xe}+\mathrm{Sn}$, measured by the INDRA collaboration [19]. This is the experiment where the most precise data are available at the moment. The SMM calculation we are discussion about are adjusted to reproduce the subset of events fulfilling the condition of completeness (more the 80the total charge and the total momentum are detected) and they show a flow angle $\theta_{f} l o w>60^{\circ}$ [19]. When comparing with a dynamical calculations, we will use the subset of events where the total transverse energy of light charged particles $E_{\text {trans }}>450 \mathrm{MeV}$. Both models, SMM and QMD, have been extensively used to interpret this reaction [19]-[21]. We would like to mention that an analysis of the QMD calculations shows that in this approach the system never passes through a state of thermal equilibrium [22] whereas the application of SMM is only justified if the fragments are formed from an equilibrized subsystem. Thus the reaction scenarios in these models are orthogonal. For the comparison with the data both calculations have been filtered with the experimental acceptance and a centrality cut is employed [21]. The increase of the average transverse energy of the fragments as a function of their mass, observed in the experiment, is larger than predicted in SMM [19] calculations. Therefore the statistical model calculations have been modified by adding a fourth system parameter, a collective energy, which is parameterized as $E_{\text {coll }}=c * A$, where $\mathrm{c}$ is a parameter which remains to be determined and $\mathrm{A}$ is the fragment mass. The best agreement the subset of experimental data fulfilling the centrality condition $\theta_{f} l o w>60^{\circ}$ and SMM calculations is obtained with the following set of input parameter:

freeze out density: $\quad 1 / 3 \rho_{0}$ 
source size: $\quad \mathrm{Z}_{S}=78 \quad \mathrm{~A}_{S}=186$

excitation energy: $\quad \mathrm{E}_{\text {thermal }}=7 \mathrm{~A} \mathrm{MeV} \quad \mathrm{E}_{\text {coll }}=2.2 \mathrm{~A} \mathrm{MeV}$

Even central collisions at intermediate energies have a less or more binary character and consequently emission close to beam or target velocity spoils the spectra of particle emission from a possible thermal source at rest in the center of mass. Therefore a meaningful comparison between statistical model calculations and experimental data is only possible around $\theta_{C M}=90^{\circ}$. We subdivide therefore the experimental data and the simulations into two equal size $2 \pi$ intervals:

$$
\begin{gathered}
B_{\text {obs }}: \quad 60^{\circ} \leq \theta_{C M} \leq 120^{\circ} \\
B_{\text {unobs }}: \quad \theta_{C M}<60^{\circ}, \theta_{C M}>120^{\circ} .
\end{gathered}
$$

In $B_{o b s}$ we observe a flat angular distribution and a constant average energy of IMF's and LCP's [20] as a function of the emission angle, both being prerequisites for a statistical equilibrium. Applying the statistical models, one assumes that in $B_{\text {unobs }}$ a preequilibrium component is superimposed to the thermal component. Therefore the statistical model cannot be compared to data there. In the dynamical model there is of course nothing like a distinction between an equilibrium and a preequilibrium part therefore we can compare the results with the data. As fig. 2 shows, QMD describes there the fragment spectra quite well [21]. Now we come to the comparison in $B_{\text {obs }}$. In fig. 3 we display on top the average kinetic energy of the fragments. On the bottom we show the charge yield distribution. We display the results for QMD and SMM calculations in comparison with the INDRA data. As one can see, these are well reproduced in both theories, underlining the above mentioned observation that these observables are not sensitive to the reaction mechanism. Hence we are confronted with the fact that completely different underlying reaction dynamics produces the same key observables for the fragments seen in $60^{\circ} \leq \theta_{C M} \leq 120^{\circ}$. Therefore more complicated observables have to be employed to distinguish between the different possible reaction mechanisms. The next more complicated is $\frac{d^{2} \sigma}{d E d A}$.

\subsection{Fragment energy spectra}

In fig. 4 , left hand side, we compare the experimental fragment kinetic energy spectra for emission at $60^{\circ} \leq \theta_{C M} \leq 120^{\circ}$ with the SMM calculation for three different fragments. On the right hand side a surprisal analysis is presented to show the structure of the deviation between the experimental and theoretical energy distribution. We see that the increase of the average fragment kinetic energy as a function of the fragment mass - seen is fig. 3 - has a quite different reason in the SMM calculations as compared to the experiment. In SMM the maximum of the energy distribution increases due to the flow but the high energy slope remains almost constant. This is expected because the disintegration due to phase space produces the same slope for all particles. In the INDRA data the increase of the average kinetic energy is due to an increase of the high energy slope of the spectra. Because the emission of fragments at midrapidity is a rare process which is logarithmically suppressed for large charges we do not have the statistics in QMD to compare in detail the kinetic energy spectra of fragments with a charge larger than 12. For higher charges the fluctuations render the analysis meaningless, for the slopes as well as for the spectra. The spectra for selected charges are presented in fig. 1 . We see a rough agreement of the high energy slope but there are too many fragments at low energy. This 


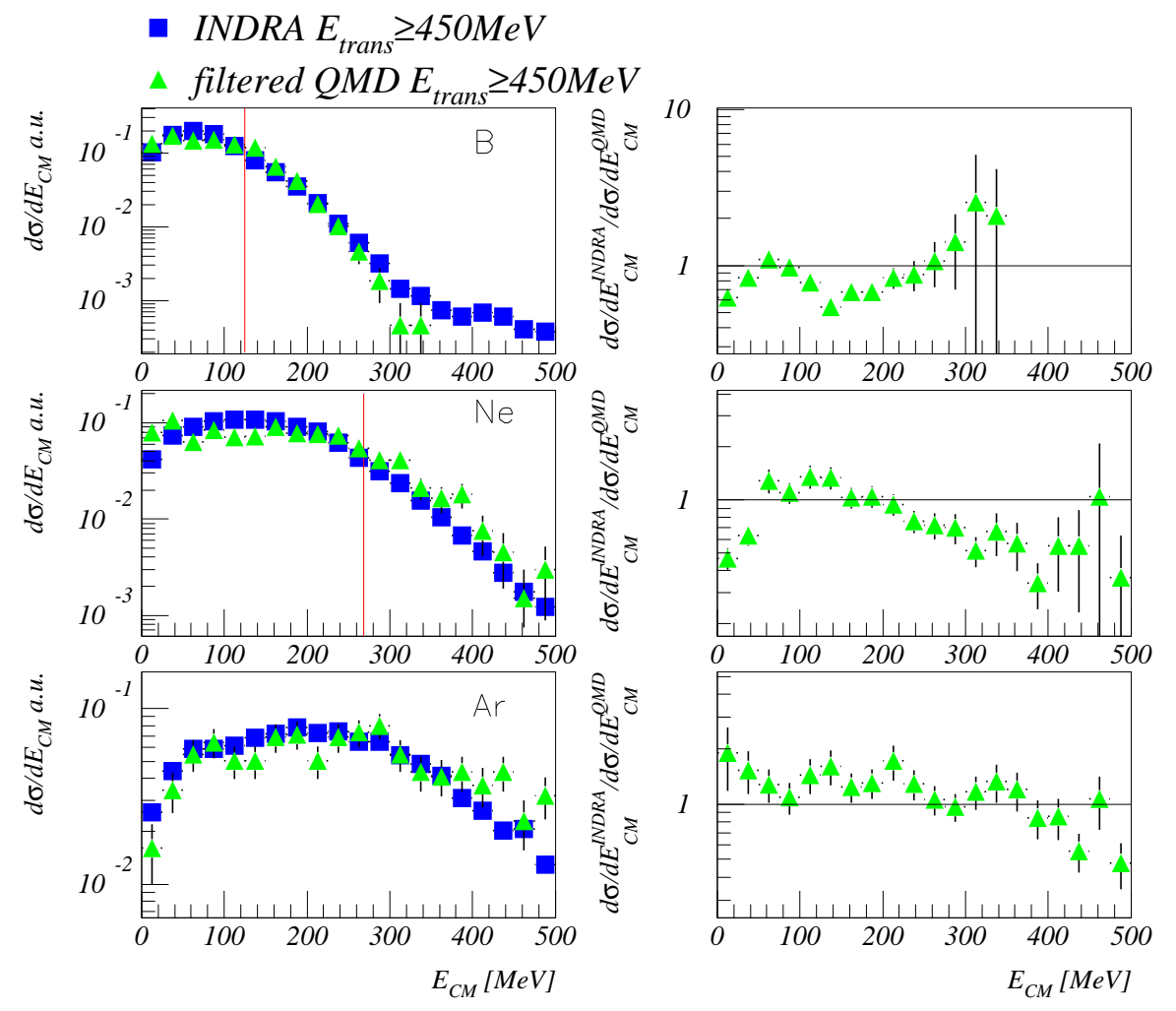

Figure 2. Central Collisions ( $E_{\text {trans }} \geq 450 \mathrm{MeV}$ ). Kinetic energy spectra of fragments emitted in forward/backward direction. The energy where fragments have $E_{\text {beam }} / N$ is marked by a line.

effect is understood [21] but there is presently no remedy to it. It is caused by the nuclear interaction range. It is too large as compared to the standard values and screens therefore a part of the repulsive Coulomb interaction. Hence one has to conclude that even the most complete experiment, available up to now, is not sufficiently precise to distinguish in a convincing way between the different theories. The more detailed the comparison is made the more the shortcomings of the approaches become apparent. As seen, precise high energy fragment data may discard the statistical models because the high energy part of the spectra has to have there a mass independent slope. To measured them is, however, a very cumbersome task. Therefore it is better to look for a more intelligent solution. A possible way to overcome this deadlock we will discuss in the next section.

In general the statistical model calculations provide excellent agreement with data as far as multiplicity distributions, correlations between the fragment masses and other quantities, which have nothing to do with the kinetic energy of the fragments, are concerned. They fail usually if they are compared with kinetic energy distributions, which show an apparent slope temperature of about $15 \mathrm{MeV}$. Such an high apparent temperature can only be created if the excitation energy/ $\mathrm{N}$ is higher than the binding energy/ $\mathrm{N}$ and hence if the fragments are not stable anymore. Hence at these excitation energies the statistical codes produce configurations consisting LCP's only. 


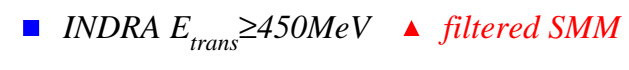

- filtered QMD $E_{\text {trans }} \geq 450 \mathrm{MeV}$
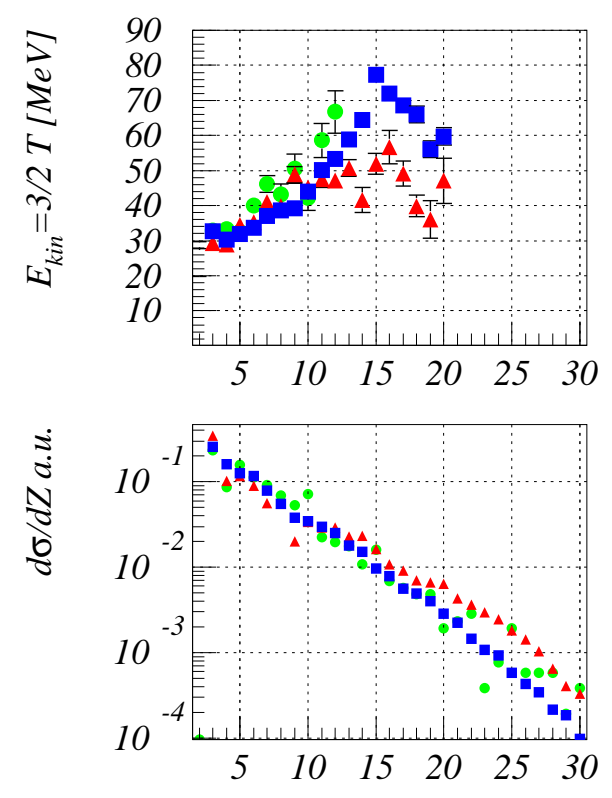

charge $Z$

Figure 3. Mean energies and charge distributions for QMD, INDRA and SMM data in $60^{\circ} \leq \theta_{C M} \leq 120^{\circ}$ for $50 \mathrm{~A} \mathrm{MeV} \mathrm{Xe}+$ Sn, central collisions.

\section{Isospin tracing- a possible method to overcome the present deadlock}

The above discussion has shown that it is all but easy to use the fragment distributions, obtained in a single reaction, to determine the reaction mechanism. Therefore it is certainly worthwhile to think about a different approach. The idea to use a combination of different projectile/target combinations to address this questions, has been advanced quite a while ago by the Texas group [24]. Changing the isospin of projectile and/or target, one may investigate the multiplicity of the different fragments and can compare the results with the prediction from a statistical model calculation which assumes that the system comes to equilibrium. The problem is, however, that the variation of the isospin is rather limited and therefore the effects are tiny. Therefore it is necessary to find a convincing analysis of the results. Recently the FOPI collaboration came up with an analysis which allows for a direct answer to the question of equilibration [25]. Following earlier work on smaller systems of the Texas group [24] they used an equal mass projectile/target combination ( $\mathrm{Ru}$ and $\mathrm{Zr}$ ) and performed all 4 possible reactions $(\mathrm{Zr}+\mathrm{Zr}$, $\mathrm{Zr}+\mathrm{Ru}, \mathrm{Ru}+\mathrm{Zr}, \mathrm{Ru}+\mathrm{Ru})$. Because the center of mass is the same many detector acceptance problems disappear, and in addition a comparison of $\mathrm{Zr}+\mathrm{Ru}$ and $\mathrm{Ru}+\mathrm{Zr}$ allows to address directly the question of equilibration as a function of the rapidity. If the system comes to equilibrium, in both combinations the same rapidity distribution is expected. If, on the contrary, there is a difference in the rapidity distribution, the assumption that the particles are emitted from a statistically equilibrated source is excluded. For the first 


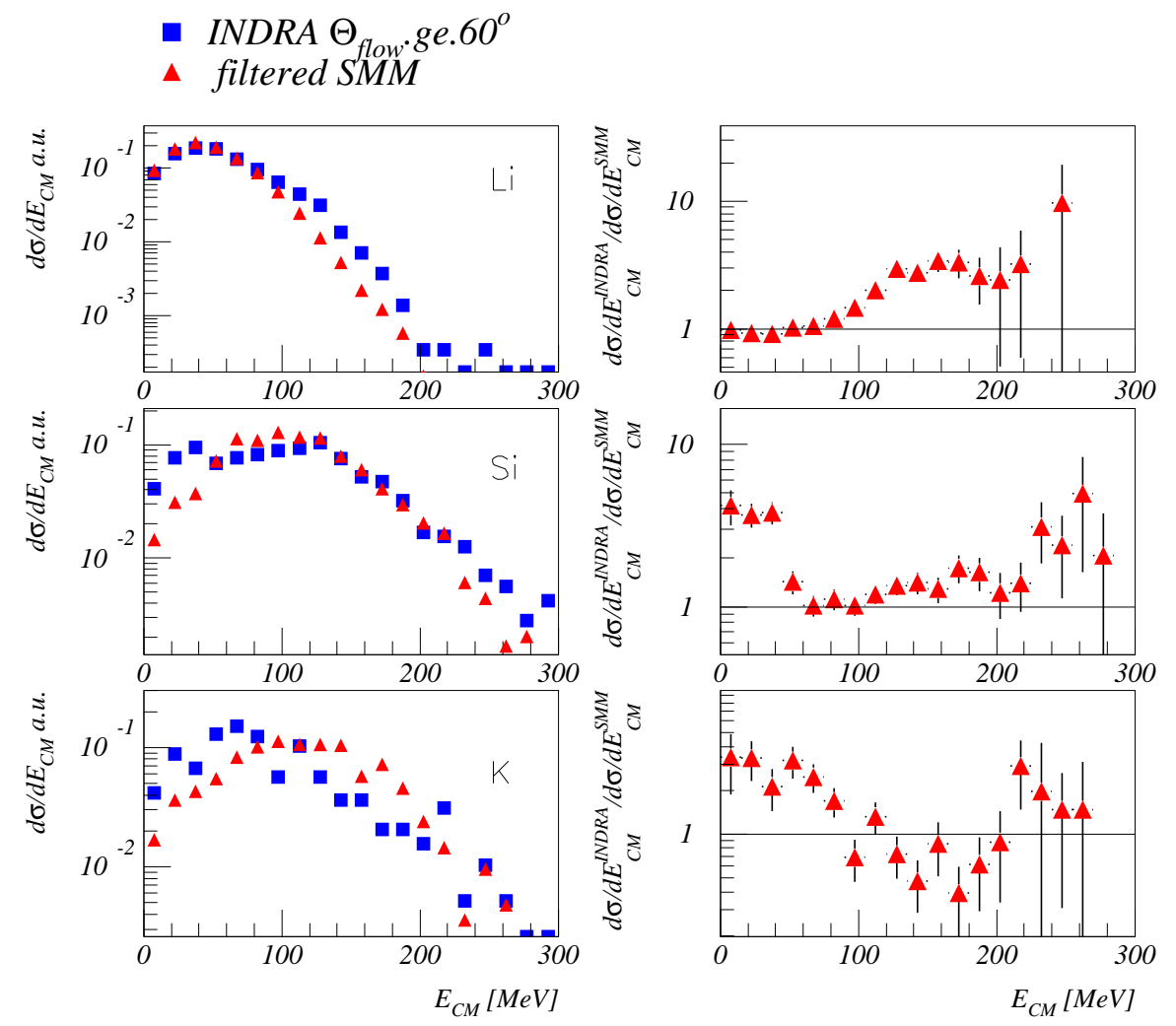

Figure 4. Emission at $60^{\circ} \leq \theta_{C M} \leq 120^{\circ}$ Energy spectra: we compare the SMM and INDRA data. On the right hand side the spectra are displayed, on the left hand side the surprisal analysis.

results of this experiment performed at $400 \mathrm{AMeV}$ we refer to the contribution of Rami. Here we only repeat the main result and discuss the interpretation in the framework of the models discussed above. In fig.6] we see $R_{Z}=\frac{2 Z-Z^{Z r}-Z^{R u}}{Z^{Z r}-Z^{R u}}$ as a function of the rapidity for the 4 different projectile/target combinations. $Z$ is the number of protons observed at the given rapidity. $R_{Z}$ gives 1 for $\mathrm{Zr}+\mathrm{Zr},-1$ for $\mathrm{Ru}+\mathrm{Ru}$ and 0 if equilibrium is reached in the mixed combinations. We see clearly that for $y^{(o)}=\frac{y}{y_{\text {beam }}}>.25$ the protons do not show a sign of equilibrium. The same has been observed for the ratio of $\mathrm{He}^{3}$ and triton [25]. Fig. 6,right, displays the result of the QMD calculations which have been performed by the FOPI-collaboration. For testing the sensitivity of the results they have employed different nucleon nucleons cross sections by rescaling the fitted experimental values by a given factor. We see that the proton rapidity distribution depends quite sensitively on the cross section. Already an increase by a factor of 2 brings the system close to equilibrium. The experimental data are best described by assuming that the cross section in the nuclear medium is close to the free one.

Although this beautiful experiment has shown that at a beam energy of $400 \mathrm{AMeV}$ the protons and the light fragments are not emitted by an equilibrated source we have no definite answer about the mechanism of multifragmentation yet. Therefore we have to continue along this proposition. We have to study the rapidity distribution of fragments not only at $400 \mathrm{MeV}$ but at lower energies as well. But this idea to use the isospin degree 


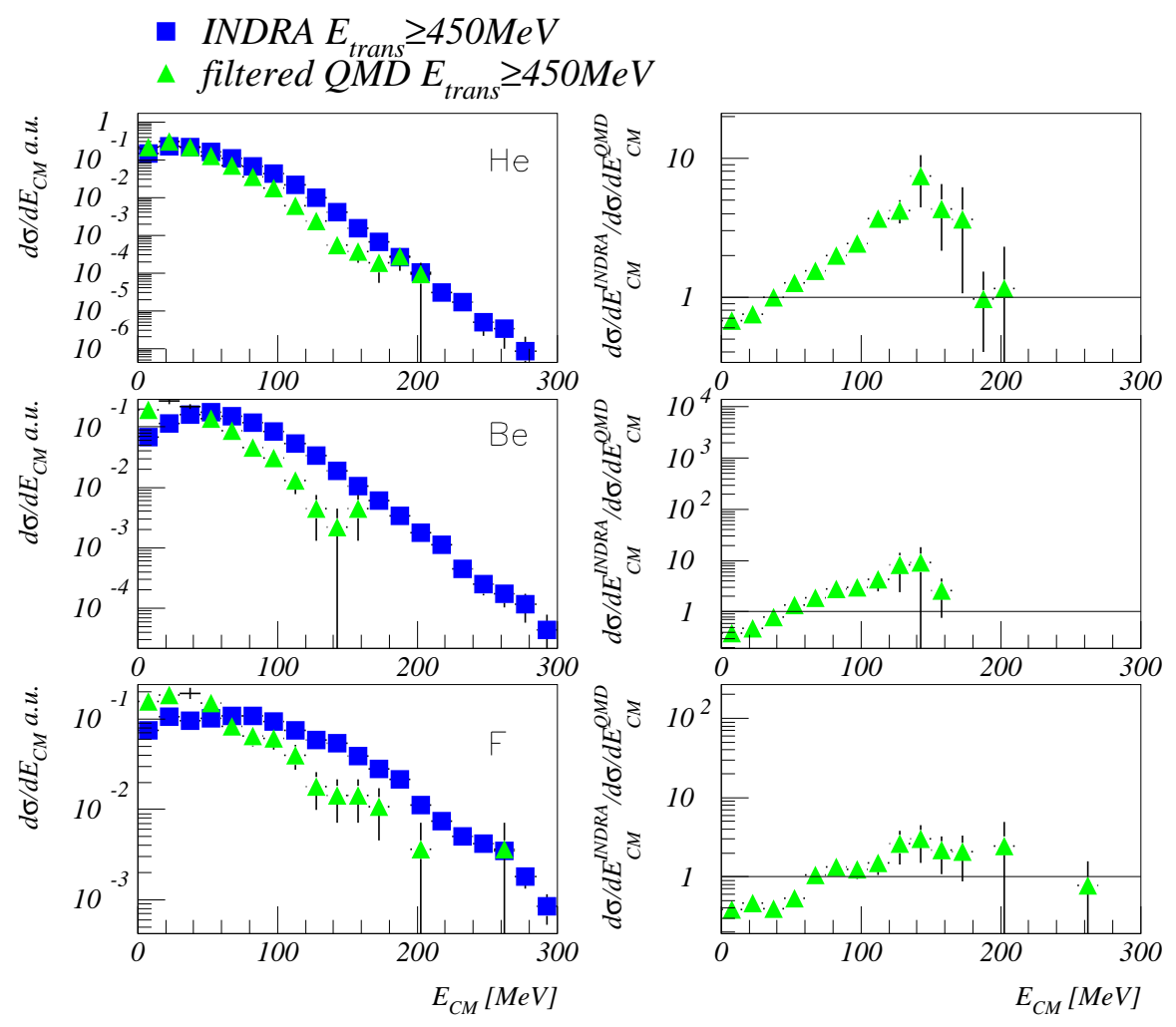

Figure 5. Emission at $60^{\circ} \leq \theta_{C M} \leq 120^{\circ}$ Energy spectra: we compare the $Q M D$ and INDRA data. On the right hand side the spectra are displayed, on the left hand side the surprisal analysis.

of freedom as a tag for equilibration, may free us from the present deadlock and may give a final answer concerning the mechanism of multifragmentation.

\section{Canonical models-applicable to nuclear physics?}

In recent times [23] it has been conjectured that the system formed in heavy ion reactions can be described by canonical thermodynamics as well. This conjecture is the basis for the caloric curve, which displays the temperature as a function of the excitation energy of the system. In case that the system suffers a first order phase transition, this function becomes flat, i.e. an increase in the excitation energy does not corresponds anymore to an increase in temperature because the energy is used as latent heat. The Aladin collaboration has claimed to have seen such a sign for a first order phase transition 23. The prerequisite for the meaningfulness of such a plot is the possibility to measure the temperature and the excitation energy at the same time. Since the temperature is extracted from the fragment yield it is the temperature of the IMF's which counts. Here we do not discuss the question how experimentally these quantities can be measured. We are concerned with the question whether for a system as small as that created in nuclear reactions the temperature fluctuations for a given excitation energy are sufficiently small to make a caloric curve possible. In a large system, the fluctuation of the excitation energy for a 


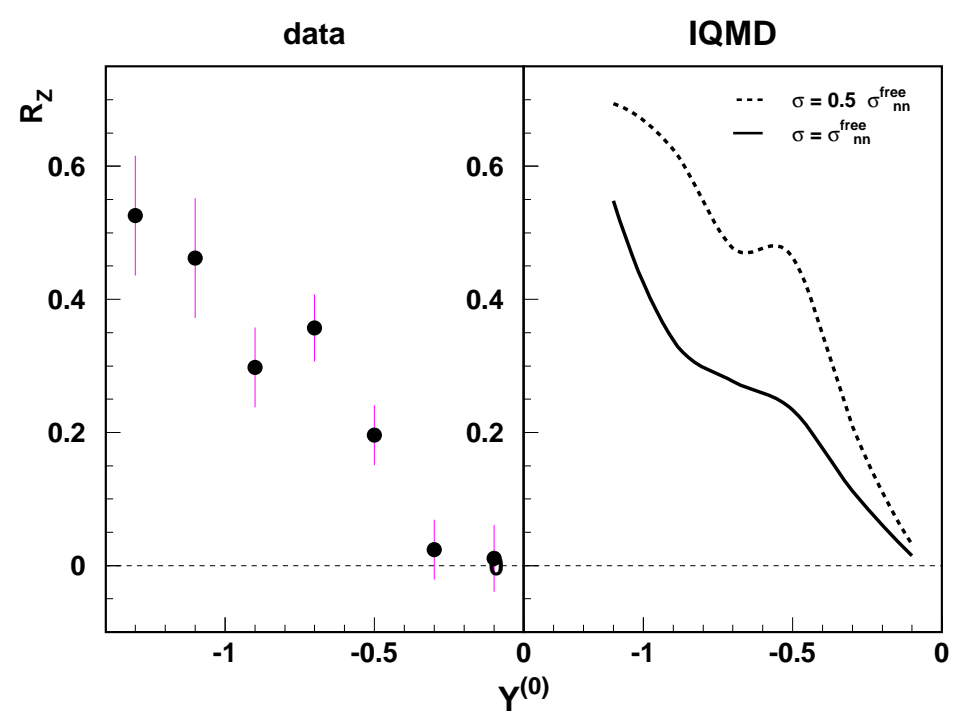

Figure 6. left: $R_{Z}$ (see text) as a function of the scaled cm rapidity for the 4 equal mass target projectile combinations. Right:QMD predictions for $R_{Z}$ (see text)for different in medium nucleon nucleon cross sections.

given temperature is $\propto \frac{1}{\sqrt{N}}$ where $N$ is the number of particles in the system. In a large system, with large $\mathrm{N}$ these fluctuations are therefore negligible and for a given excitation energy we find a precise value for the temperature of the system.

In order to discuss this question we would like to start with an explanation of the differences between the microcanonical description of a system, as done in the statistical multifragmentation models, and canonical thermodynamics. The basis of all statistical mechanics is the counting of microstates. To make life easier we take a concrete example. Consider an harmonic oscillator whose energy levels i $(i \geq 0)$ can be occupied by particles which have energies $\epsilon_{i}=\hbar \omega \cdot i$ We have suppressed the zero point motion here. The total energy of the particles is given by $E=\sum \epsilon_{i} \cdot n_{i}$ where $n_{i}$ is the number of particles which are on the level i. Now we can calculate the number of different repartition which exists for a given number of particles $N=\sum n_{i}$ and a given energy $E=\sum \epsilon_{i} n_{i}$. For example, if we have $\mathrm{N}=2$ and $E=2 \hbar \omega$ we can either have both particles on the level $\mathrm{i}=1$ or one particle on the level $\mathrm{i}=0$ and the other on $\mathrm{i}=2$. Each of these repartitions is called a microstate. $g(N, E)$ is the number of microstates for a given E,N and $\Omega(N, E)=k \ln g(N, E)$ is the entropy of the system ( $\mathrm{k}$ is the Boltzmann constant).

Now, assume that we have two oscillators which can transfer energy and particles and have the total energy $E=E_{1}+E_{2}$ and the total particle number $N=N_{1}+N_{2}$. The number of microstates of the combined system is given by

$g(N, E)=\sum_{E_{1}, N_{1}} g_{2}\left(N_{1}, E_{1}\right) g_{1}\left(N-N_{1}, E-E_{1}\right)$

where $g_{1}\left(E_{1}, N_{1}\right)$ and $g_{2}\left(N_{2}, E_{2}\right)$ are the number of microstates of the oscillators 1 and 2 for given energies and particle numbers $E_{1}, N_{1}, E_{2}, N_{2}$, respectively.

The temperature is defined as $\frac{k \partial l n g}{\partial E}$. For our combined oscillators

$g\left(N, E, E_{1}\right)=\sum_{N_{1}} g_{2}\left(N_{1}, E_{1}\right) g_{1}\left(N-N_{1}, E-E_{1}\right)$ 
one finds

$$
\begin{aligned}
\frac{k \partial \ln g\left(N, E, E_{1}\right)}{\partial E_{1}} & =\sum_{N_{1}} \frac{k \partial \operatorname{lng}_{1}\left(N_{1}, E_{1}\right)}{\partial E_{1}}+\frac{k \partial \operatorname{lng}_{2}\left(N-N_{1}, E-E_{1}\right)}{\partial E_{1}} \\
& =\sum_{N_{1}} \frac{\partial k \operatorname{lng}_{1}\left(N_{1}, E_{1}\right)}{\partial E_{1}}-\frac{\partial k \operatorname{lng} g_{2}\left(N-N_{1}, E_{2}\right)}{\partial E_{2}} \\
& =\sum_{N_{1}} \frac{1}{T_{1}}-\frac{1}{T_{2}}
\end{aligned}
$$

What can one conclude? Only if $g\left(N, E, E_{1}\right)$ is maximal (at $E_{1}=E_{1}^{\max }$ ) the temperatures in the two subsystems are identical. Otherwise the temperatures are different. Hence for small systems it is the generic case that both subsystems have different temperatures. This is completely counterintuitive because our experience is based on the large systems we encounter in real life where - in equilibrium - the two subsystems have the same temperature. There the sum in eq. 2 can be very well approximated by the largest term

$$
\sum_{N_{1}} g_{2}\left(N_{1}, E_{1}\right) g_{1}\left(N-N_{1}, E-E_{1}\right) \approx g_{2}\left(N_{1}, E_{1}^{\max }\right) g_{1}\left(N-N_{1}, E-E_{1}^{\max }\right)
$$

Only if this approximation is justified it makes sense to describe the system as a canonical system, i.e. in the variables $\mathrm{T}$ and $\mu$. Then the system obeys all the laws of equilibrium thermodynamics, that the entropy becomes additive and that subsystems in equilibrium have the same temperature and the same chemical potential.

Is this approximation justified in heavy ion collisions where the systems are still rather small containing at most around 400 particles? We use the above mentioned microcanonical SMM calculations to find an answer. We identify the two subsystems with the fragments with $Z \geq 3$ and the LCP's, respectively, and calculate the temperature difference between the subsystems.

In fig. 7, top, we display the number of events (left) and the average number of IMF's (right) as a function of the total number of nucleons entrained in the fragments and the total fragment energy. We see a rather broad distribution. In the middle we present the difference of the temperature and the chemical potential in the two subsystems (LPC's and IMF's)as a function of the total mass and the total energy of all IMF's. For calculating the temperature difference we have assumed that $\frac{1}{T_{1}}-\frac{1}{T_{2}} \approx \frac{\Delta T}{T^{2}+\Delta T^{2} / 4}$ where $T$ is assumed to be $7 \mathrm{MeV}$. We see a rather broad distribution of the chemical potentials and of the temperatures. The probability distribution of fragments to be emitted in a microstate which shows a $|\Delta T|$ and a chemical potential difference of $|\Delta \mu|$, respectively, between the two subsystems is plotted in the bottom row. The chemical potential $|\Delta \mu|$ in units of the temperature fluctuates by about to $40 \%$ around the mean value 0 whereas the variation of the distribution of the temperature difference between the subsystems is about $1.7 \mathrm{MeV}$. More precisely the temperature in the subsystem of the fragments cannot be determined. Consequently, the temperature values in the caloric curve have to get an error bar of this size which renders any conclusion about a phase transition useless because one cannot distinguish whether $\mathrm{T}$ increases slowly as a function of the excitation energy (cross over) or whether it stays constant (first order phase transition).

This variance of the temperature in the subsystems renders as well the application of canonical thermodynamics to heavy ion reaction useless. There is nothing like a well 
defined temperature of the matter created in these reactions and therefore the hope to measure the system temperature is in vain.
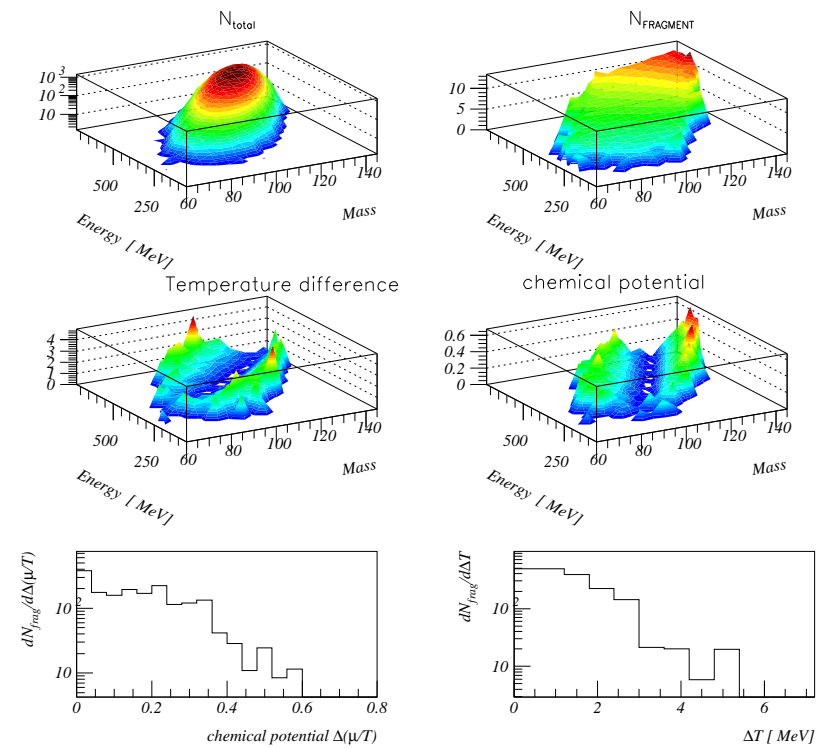

Figure 7. Top: Number of events and number of fragments as a function of the total mass of all fragments and of their total energy. Middle: Distribution of the temperature difference and the difference of the chemical potential. Bottom: Distribution of the absolute value of the temperature difference distribution and that of the distribution of the difference of the chemical potentials between the subsystems formed by fragments and LPC's, respectively, for a SMM calculation adjusted to describe the INDRA results

\section{REFERENCES}

1. J.P. Bondorf, A.S. Botvina, A.S. Iljinov, I.N. Mishustin, K. Sneppen, Phys. Rep. 257 133 (1995)

2. D.H.E Gross, Rep. Prog. Phys. 53, 605 (1990), and references therein.

3. D. Hahn and H. Stöcker, Nucl. Phys. A 476, 718 (1988)

4. , J. Tke, D.K. Agnihotri, W. Skulski, and W.U. Schrder, Phys. Rev.C accepted, appears in august 2000.

5. D.H.E Gross and K. Sneppen, Nucl. Phys. A 567, 317 (1993)

6. J. Aichelin, Phys. Rep. 202, 233 (1991), and references therein.

7. C. Hartnack et al. Eur. Phys. J. A 1 (1998) 151

8. W. Müller, M. Begemann-Bleich and J. Aichelin Phys. Lett. B 298, 27 (1993)

9. A. Bohnet, J. Aichelin, J. Pochodzalla, W. Trautmann. G. Peilert, H. Stöcker, and W. Greiner, Phys. Rev. C 44 (1991) 2111

10. M. Begemann-Bleich et al. Phys. Rev. C 48, 610 (1993)

11. P.B. Gossiaux, R. Puri, C. Hartnack and J. Aichelin Nucl. Phys. A619 (1997) 379 
12. P.B. Gossiaux and J. Aichelin Phys. Rev. C56 (1997) 2109

13. see www-subatech-in2p3.fr/ theo/qmd

14. A.S. Goldhaber, Phys. Lett. 53B (1974) 306

15. M.E. Fischer, Physics 3, 255 (1967)

C.B. Chitwood et al. Phys. Lett. B131 , 2897 (1983)

16. J. Huefner, Phys. Lett. B 173, 373 (1986)

17. J. Aichelin, J. Hüfner and R. Ibarra, Phys. Rev. C C30, 107 (1984).

18. W. Bauer, these proceedings

19. S. Salou, Thesis, U Caen, GANIL T 9706

20. N. Marie et al. Phys. Lett. B 391 (1997) 15

21. R. Nebauer et al. Nucl. Phys. A658, 67 (1999)

22. R. Nebauer et al. Nucl. Phys. A650, 65 (1999)

23. J. Pochodzalla et al.' Phys. Rev. Lett. 751040 (1995)

24. H. Johnston et al., Phys. Rev C56, 1972 (1997)

E. Ramakrishnan et al., Phys. Rev C57, 1803 (1998)

25. F. Rami et al., Phys. Rev. Lett 84, 1120 (2000) 